\title{
A surprising case of a biliary tumor-like lesion
}

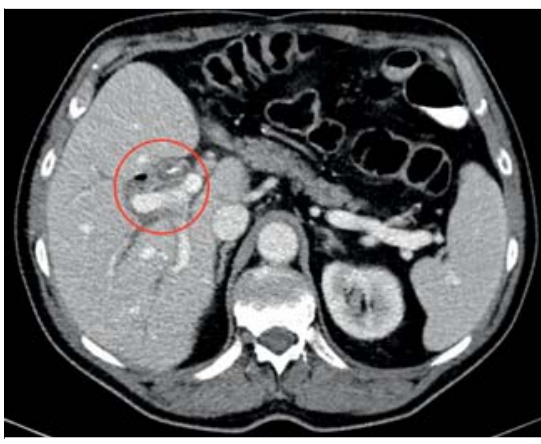

- Fig. 1 Computed tomography scan showing dilation of intrahepatic bile ducts and nodular hilar tissue (red circle).

A 74-year-old man referred to our institute complained of asthenia and jaundice associated with altered laboratory liver tests. The patient's history included cardiopathy, dyslipidemia, arterial hypertension, and partial gastrectomy with Billroth II reconstruction due to a peptic ulcer. Abdominal ultrasound showed dilation of intrahepatic bile ducts associated with hepatic hilar tissue, strongly suspected for hilar tumor (Klatskin type IV according to Bismuth-Corlette classification) [1-3] on computed tomography $(\mathrm{CT})$ scan ( $\triangleright$ Fig. $\mathbf{1}$ ), associated with low grade intra-abdominal fluid. Neoplastic markers (carbohydrate antigen 19-9, carcinoembryonic antigen, and alpha-fetoprotein) were negative. Percutaneous transhepatic-cholangiography with brushing and biliary internalexternal 10-Fr drainage placement was considered the best option. Cytology examination was inconclusive.

A post-procedural intrahepatic bleed from a pseudo-aneurysm of a branch of the hepatic artery caused an intrahepatic hematoma, solved by arterial embolization and percutaneous drainage ( Fig.2). Later we performed an antegrade cholangioscopy through the percutaneous access. Cholangioscopy showed a papillary, friable, and angiogenetic tissue at the hilar site, which was sampled. Surprisingly, histologic examination

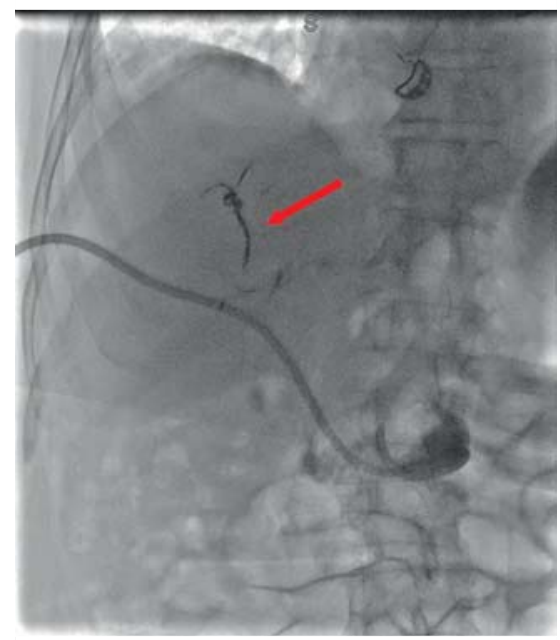

- Fig. 2 Radiological imaging of internalexternal percutaneous biliary drainage. Arterial embolization (arrow) to treat the post-procedural active bleeding from a pseudo-aneurysm of the hepatic artery.

showed only inflammatory tissue. A second percutaneous cholangioscopy was performed in the radiologic theater ( Fig.3) and an unexpected image appeared: a hard, brownish, oblong 17-mm formation was embedded in the suspected papillary tissue, so it was gently ex-
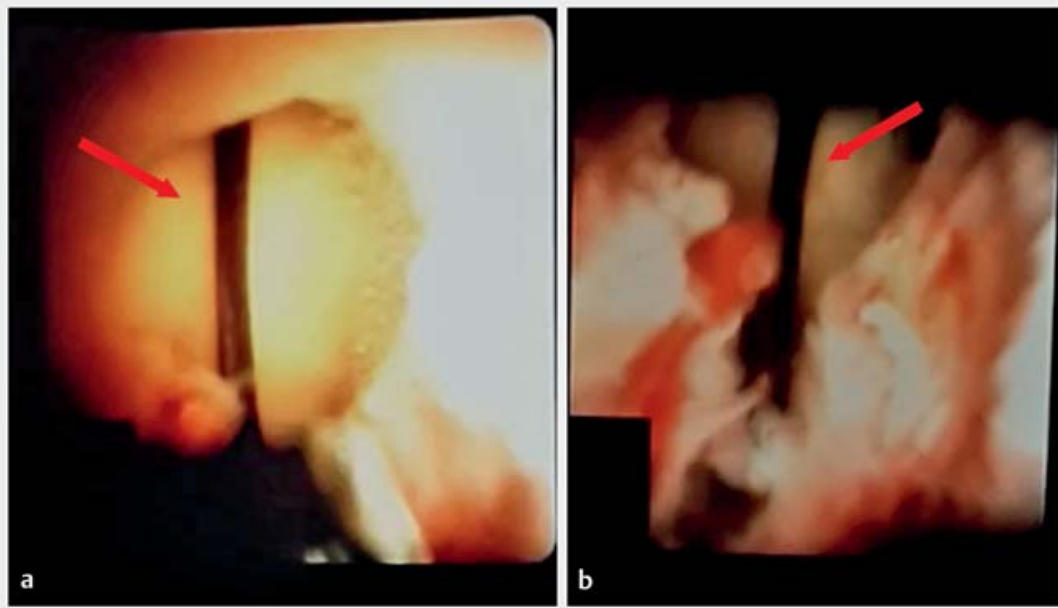

Fig. 4 a, b Endoscopic views of the hard, brownish, and oblong foreign body (arrows) embedded in the papillary hilar tissue.

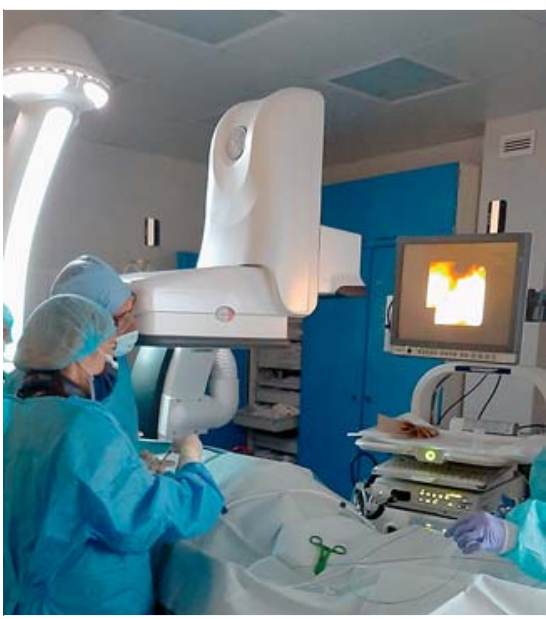

Fig. 3 Percutaneous cholangioscopy performed in the radiological theater.

tracted using a forceps for foreign bodies (• Fig.4, Video 1). On macroscopic evaluation it appeared as a fishbone; the histology showed an animal origin (vimentin dye negative) and mineral consistency comparable to a chicken bone ( $\vee$ Fig.5). The histology on the hilar tissue identified inflammatory tissue without any sign of dysplasia/neoplasia, compatible with a foreign body reaction. The pa- 


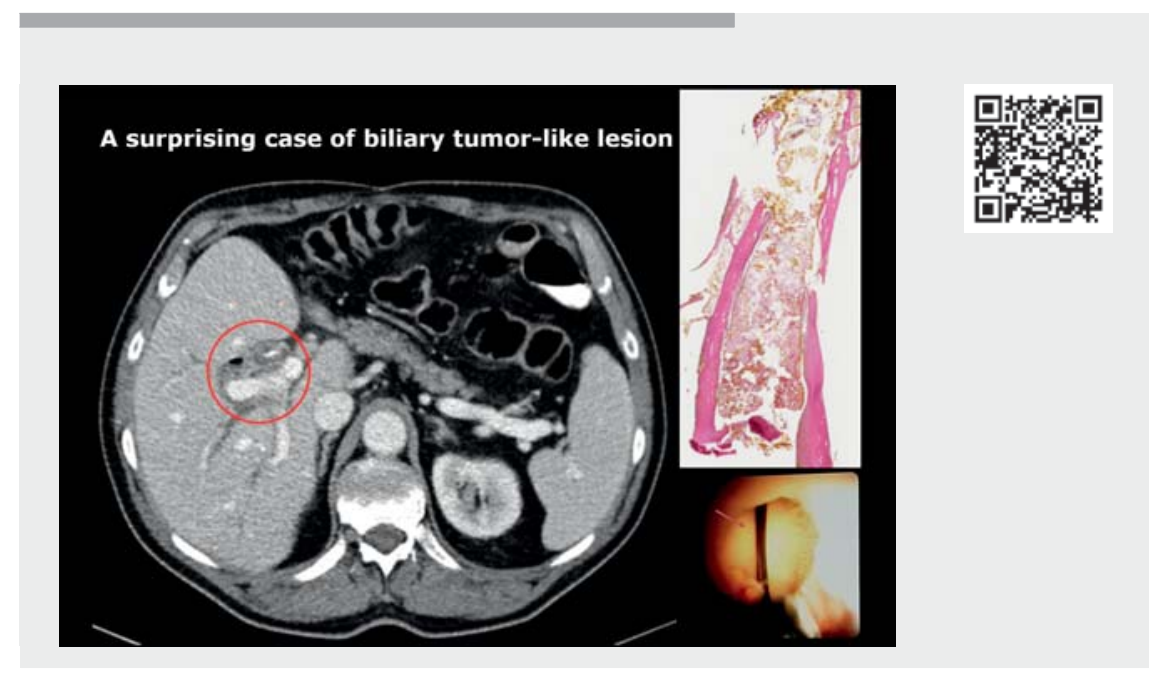

$\checkmark$ Video 1 Video showing how percutaneous cholangioscopy with biopsy is fundamental in differential diagnosis of hilar biliary tumors. A foreign body was identified and removed with forceps. The tissue covering the foreign body was sampled and the histology examination showed a mineral consistency comparable to a chicken bone.
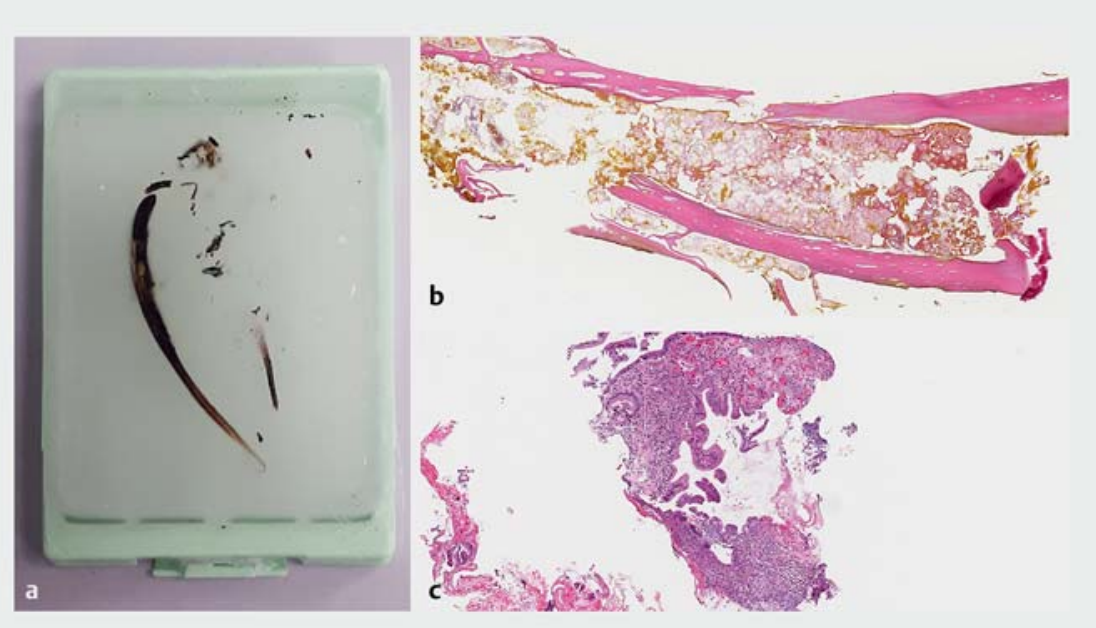

Fig. 5 a Fishbone-shaped foreign body after extraction. b Hematoxylin staining at $40 \times$ magnification showing an animal origin and a mineral consistency. c $80 \times$ magnification showing granular tissue adhered to the foreign body.

tient fully recovered after removal of biliary drainage, and the cholangiography showed a more regular hilar biliary tree with normal bile and contrast outflow. After 6 months of follow-up the patient did not complain about any other symptom and imaging did not show signs of malignancy.

Endoscopy_UCTN_Code_TTT_1AR_2AK

\section{Competing interests}

The authors declare that they have no conflict of interest.

The authors

\section{Giacomo Emanuele Maria Rizzo ${ }^{1,2,3}$ @ Luigi} Maruzzelli ${ }^{4}$, Rosa Liotta ${ }^{5}$, Roberto Miraglia ${ }^{4}$, Salvatore Gruttadauria ${ }^{6,7}$, Mario Traina ${ }^{1}$, Ilaria Tarantino ${ }^{1} \odot$

1 Endoscopy Service, Department of Diagnostic and Therapeutic Services, IRCCSISMETT, Palermo, Italy
2 Department of Surgical, Oncological and Oral Sciences (Di.Chir.On.S.), University of Palermo, Palermo, Italy

3 Section of Gastroenterology \& Hepatology, Department of Health Promotion Sciences Maternal and Infant Care, Internal Medicine and Medical Specialties, PROMISE, University of Palermo, Palermo, Italy

4 Radiology Unit, Department of Diagnostic and Therapeutic Services, IRCCS-ISMETT, Palermo, Italy

5 Pathology Unit, Department of Diagnostic and Therapeutic Services, IRCCS-ISMETT, Palermo, Italy

6 Department for the Treatment and Study of Abdominal Diseases and Abdominal Transplantation, IRCCS-ISMETT (Istituto di Ricovero e Cura a Carattere Scientifico Istituto Mediterraneo per i Trapianti e Terapie ad alta specializzazione), UPMC (University of Pittsburgh Medical Center), Palermo, Italy

7 Department of Surgery and Medical and Surgical Specialties, University of Catania, Catania, Italy

\section{Corresponding author}

\section{Ilaria Tarantino, MD}

Endoscopy Service, Department of Diagnostic and Therapeutic Services, IRCCS-ISMETT Palermo, Via Ernesto Tricomi 5, 90127 Palermo, Italy itarantino@ismett.edu

\section{References}

[1] Klatskin G. Adenocarcinoma of the hepatic duct at its bifurcation within the porta hepatis. An unusual tumor with distinctive clinical and pathological features. Am J Med 1965; 38: 241-256

[2] Bismuth H, Corlette MB. Intrahepatic cholangioenteric anastomosis in carcinoma of the hilus of the liver. Surg Gynecol Obstet 1975; 140: 170-178

[3] Nakeeb A, Pitt HA, Sohn TA et al. Cholangiocarcinoma. A spectrum of intra-hepatic, perihilar, and distal tumors. Ann Surg 1996; 224: 463-475

\section{Bibliography}

Endoscopy 2022; 54: E717-E718

DOI 10.1055/a-1759-2377

ISSN 0013-726X

published online 7.3.2022

(c) 2022. Thieme. All rights reserved.

Georg Thieme Verlag KG, Rüdigerstraße 14,

70469 Stuttgart, Germany 